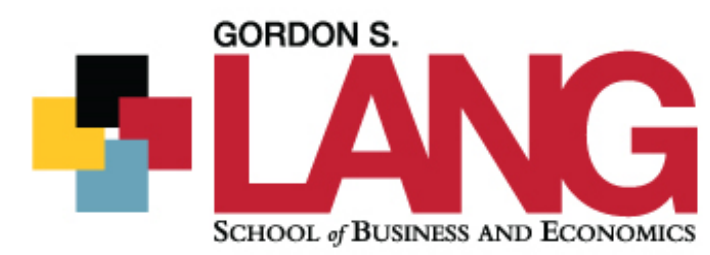

Department of Economics and Finance

Gordon S. Lang School of Business and Economics

University of Guelph

Discussion Paper 2020-01

A principal component-guided sparse

regression approach for the determination of

bitcoin returns

By:

Thanasis Stengos

University of Guelph

tstengos@uoguelph.ca
Theodore Panagiotidis

University of Macedonia tpanag@uom.gr

\author{
Orestis Vravosinos \\ New York University \\ orestis.vravosinos@nyu.edu
}




\title{
A principal component-guided sparse regression approach for the determination of bitcoin returns
}

\author{
THEODORE PANAgIOTIDIS \\ Thanasis Stengos \\ University of Macedonia, Greece \\ University of Guelph, Canada \\ Orestis VRavosinos \\ New York University, USA
}

January 12020

\begin{abstract}
We examine the significance of fourty-one potential covariates of bitcoin returns for the period 2010-2018 (2,872 daily observations). The principal component-guided sparse regression is employed, introduced by Tay et al. (2018). We reveal that economic policy uncertainty and stock market volatility are among the most important variables for bitcoin. We also trace strong evidence of bubbly bitcoin behavior in the 2017-2018 period.
\end{abstract}

Keywords: bitcoin; cryptocurrency; bubble; sparse regression; LASSO; PC-LASSO; principal component; flexible least squares

JEL classification codes: G12; G15 


\section{INTRODUCTION}

$\mathrm{I}^{\mathrm{N}}$ NTRODUCED by Nakamoto (2008) bitcoin is a digital currency with characteristics not found in traditional currencies-for example it is not as robust a medium of exchange or store of value as traditional currencies. As outlined by Panagiotidis et al. (2018b), it allows for transactions without bank intermediation or any transaction fees, and also offers anonymity. To get a sense of the pace in which the overall frequency of bitcoin transactions has been increasing, note that from less than 10,000 daily bitcoin transactions in the period 2011-2012, in 2019 there have been around 300,000 bitcoin transactions per day. ${ }^{1}$

We examine the potential covariates of bitcoin returns (e.g., stock market returns, stock market volatility, exchange rates, commodities, central bank rates, internet trends and policy uncertainty). Panagiotidis et al. (2018a) have also considered a variety of potentially important variables for bitcoin returns (21 variables in total whereas here we consider 41) employing the Least Absolute Shrinkage and Selection Operator (LASSO). We examine a larger set of potential variables (41) and account for the group structure of the independent variables.

We employ the principal component-guided sparse regression (PC-LASSO) introduced by Tay et al. (2018). We identify the variables that are most important for bitcoin. This procedure allows us to consider numerous potential variables (41 in our case) and select only a subset of the covariates (as standard LASSO does). We thus consider far more variables possibly important for bitcoin than in earlier studies (e.g., Panagiotidis et al., 2018a,b). PC-LASSO also exploits the correlation and the group structure of the independent variables by shrinking each group-wise component of the solution towards the leading principal components of that group (e.g., the group of stock market returns variables), which is not the case for standard LASSO employed in Panagiotidis et al. (2018a). Doing so it yields results contradicting some of those in Panagiotidis et al. $(2018 \mathrm{a}, \mathrm{b})$. Next, we perform a rolling-window PC-LASSO estimation to gauge how the interaction of alternate variables with bitcoin has changed over time. Last, we examine their variability by keeping only the variables surviving the PC-LASSO (i.e., that have non-zero coefficients) and employing the Flexible Least Squares (FLS) methodology of Kalaba and Tesfatsion (1989). The rest of the paper is organized as follows: Section 2 presents the data and methods used, Section 3 discusses the results and the last section concludes.

\footnotetext{
${ }^{1}$ See www.blockchain.com/en/charts/n-transactions?timespan=all.
} 


\section{Methods ANd DATA}

We use daily (7-day week) data for the period spanning from July 21st 2010 to May 31st 2018 (2,872 observations). Most of the data come from Thomson Reuters Eikon, while for bitcoin the Coindesk Bitcoin Price Index (BPI) is used. Wikipedia trend data were retrieved using the package 'wikipediatrend' till the 21st of January 2016 (they ere not available after this date). More recent data were filled from tools.wmflabs.org. ${ }^{2}$ The reader is referred to Table 4 in the Appendix for a complete list of the independent variables considered.

The variables that are not in daily frequency or are in a 5-day week frequency have been linearly interpolated. ${ }^{3}$ Variables are used in levels or first differences depending on their stationary characteristics ${ }^{4}$ For comparability of the coefficients, all variables are standardized to have mean 0 and a variance of 1 . As in Forbes and Rigobon (2002), we account for differences in opening hours of the stock markets by using 2-day rolling averages for stock-market specific variables.

Similar to Panagiotidis et al. (2018a), we consider the full sample, as well as three sub-periods of it separately: (1) July 21st 2010 - December 10th 2013, (2) December 11th 2013 - March 24th 2017 and (3) March 25th 2017 - May 31st 2018. The selection of these three periods is motivated by (1) the different phases the bitcoin market has been through and (2) the rolling window generalized supremum ADF test for bubbles (GSADF; Phillips et al., 2015) (see Figure 2 in the Appendix). ${ }^{5}$ The first period reflects the early phase of bitcoin with lower traded quantities including the first bitcoin boom in late 2013 and Mt. Gox's suspension of trading and filing for bankruptcy protection. The second is a period of higher stability and gradual recovery, while the third one corresponds to the recent alleged bubble. ${ }^{6}$ The selection of the break points is corroborated by

\footnotetext{
${ }^{2}$ Due to discrepancies in the data and different scaling between the two sources, a simple linear regression was estimated for the time period for which data from both sources was available and Wikipedia trend values from 21 January and on were estimated using the values from tools.wmflabs.org.

${ }^{3}$ Google does not provide trend data in daily frequency for large time periods. Thus, for higher precision daily Google trend data were obtained in nine-month intervals, then log differences were computed and the values for the missing observations every nine months were interpolated.

${ }^{4}$ All variables were found to be $\mathrm{I}(0)$. Unit root tests are available upon request.

${ }^{5}$ GSADF is implemented in Eviews (see Caspi, 2017). Although in their empirical application Phillips et al. (2015) use S\&P 500 stock price index and the real S\&P 500 stock price index dividend data to implement the test on the price-dividend ratio, in our case it is hard to argue for any (measure of) fundamental value of bitcoin, so we implement the test on bitcoin alone. Looking into the fundamental drivers of the Bitcoin and Ethereum price, Corbet et al. (2018) test for the existence and dates of pricing bubbles employing the earlier methodology introduced in Phillips et al. (2011).

${ }^{6}$ See for example ft.com/1, ft.com/2, and ft.com/3.
} 
the sub-periods obtained in Panagiotidis et al. (2018a) under different methods, namely an ADF Breakpoint unit root test.

We estimate PC-LASSO models for each of the three periods and the entire sample. ${ }^{7}$ This is done with two alternative approaches: first with the principal component (PC) groups defined by different market/factor types (i.e., stock market returns, policy uncertainty) and second, with the PC groups defined by geographical region (i.e., US, Europe etc.). PC-LASSO admits overlapping PC groups, which allows us to ascribe variables to two different geographical regions when necessary. With the first approach for PC grouping, we gain insight on which variables are the most important for bitcoin within each market/factor type, while with the second which variables are the most significant within each geographical region. Next, we perform a rolling-window PC-LASSO estimation to gauge how the sign and the importance of the interaction of alternate variables with bitcoin has changed over time.

Tay et al. (2018) describe PC-LASSO as a method for supervised learning combining the LASSO sparsity penalty with a quadratic penalty that shrinks the coefficient vector toward the leading principal components of the independent variables. When the independent variables can be grouped to different categories, it shrinks each group-wise component of the solution toward the leading principal components of that group. PC-LASSO is discussed in section A in the Appendix.

Using the variables that have a non-zero coefficient in the PC-LASSO with market/factor type PC groups for the full sample, we estimate a time-varying linear regression employing the FLS approach of Kalaba and Tesfatsion (1989) with Kalman filtering and check the robustness of the results using a standard state-space approach where coefficients are treated as separate random walks. ${ }^{8}$

Table 1 summarizes the approaches employed in the paper.

\section{REsUlts}

In line with Panagiotidis et al. $(2018 \mathrm{a}, \mathrm{b})$, variables such as economic policy uncertainty and stock markets volatility emerge as the most important ones featuring a negative relation with bitcoin. We find foreign exchange (FX) markets, monetary policy and popularity measures to be of relatively minor importance. More profound was the effect of traditional stock market returns corroborating the evidence in Panagiotidis et al. (2018b). The US stock market emerges as the most important one-in terms of both volatility and returns; the positive relationship

\footnotetext{
${ }^{7}$ We implement PC-LASSO using the R package 'pcLasso'. Hotz-Behofsits et al. (2018) test the forecasting performance of sparse state space models using a limited number of predictors.

${ }^{8}$ The FLS and state-space methods are implemented using the add-in 'topuni' in Eviews. For the results see section B in the Appendix
} 
Table 1: Summary of methods used

\begin{tabular}{|c|c|}
\hline Method & Details - specifications \\
\hline GSADF & $\begin{array}{l}\text { Initial window size: } 100 \text { obs; intercept and trend included in the } \\
\text { equation; number of lags in the test equation selected based on the } \\
\text { Schwarz information criterion (with maximum allowed 7) }\end{array}$ \\
\hline PC-LASSO & $\begin{array}{l}\text { Dependent variable distribution: Gaussian; } r=0.7 \text {; principal } \\
\text { component groups as given in the market/factor type column (Table } 2 \\
\text { results) or in the region column (Table } 3 \text { results) of Table } 4 ; \lambda \text { selected } \\
\text { through } 30 \text {-fold cross validation (that is, by repeated estimations of the } \\
\text { model in subsamples of the total sample and making out-of-sample } \\
\text { predictions, we find which value of } \lambda \text { leads to better predictions on } \\
\text { average); standardized variables; convergence threshold for coordinate } \\
\text { descent algorithm: } 10^{-4}\end{array}$ \\
\hline $\begin{array}{l}\text { Rolling } \\
\text { window } \\
\text { PC-LASSO }\end{array}$ & $\begin{array}{l}\text { Dependent variable distribution: Gaussian; } r=0.7 ; \lambda=15 \text {; window } \\
\text { size: } 200 \text { obs.; principal component groups as given in the } \\
\text { market/factor type column of Table } 4 \text {; variables standardized in each } \\
\text { window separately so that they are comparable within each time } \\
\text { window; convergence threshold for coordinate descent algorithm: } 10^{-4}\end{array}$ \\
\hline FLS & $\begin{array}{l}\text { Flexible Least Squares (Kalaba and Tesfatsion, 1989) and state-space } \\
\text { models with Kalman filtering; smoothing parameter set equal to } 1 / 0.1\end{array}$ \\
\hline
\end{tabular}

Note: GSADF and PC-LASSO have been proposed by Phillips et al. (2015) and Tay et al. (2018), respectively.

with US stock market returns and the negative one with volatility point to some degree of connection between bitcoin and traditional financial markets. Interestingly, even though popularity measures (i.e., Google and Wikipedia article access trends) appear not to be significant overall, in the second sub-period-that is, after the first bitcoin boom and Mt. Gox's suspension of trading and filing for bankruptcy protection-both variables are negatively related to bitcoin returns. This contradicts the results of Panagiotidis et al. $(2018 a, b)$ who find a positive and more pronounced consistent link between Google trends and bitcoin returns. Still in contrast to these results, we find gold not to play a role, which also holds for the other commodities considered. Also, EU monetary policy only appears to have played a role at the early stages of the European debt crisis, while their results suggested a stronger role of the ECB rate. Last, government bond yields seem to matter for bitcoin.

Table 2 presents the PC-LASSO coefficients for the full sample and for each of the three sub-periods separately when PC groups are formed by market/factor 
type. The CBOE Volatility Index is the most important suggesting that among the US, Europe and Japanese stock markets, the volatility of the US one is the most significant for bitcoin.

Table 3 presents the PC-LASSO coefficients for the full sample and for each of the three sub-periods separately when PC groups are formed by geographic region. Again the CBOE Volatility Index appears to be among the most relevant variables for bitcoin and among the US variables. Russia economic policy uncertainty (EPU) is with both PC groupings negatively associated to bitcoin returns. Notice also that in the first era of bitcoin, economic policy uncertainty is crucial for bitcoin. Last, under both PC groupings all variables but the US Fed Funds effective rate (FFER) have a zero coefficient in the most recent period. This combined with the GSADF statistic (Figure 2) serves as signal of bubbly behavior in the period 2017-2018 with bitcoin weakly connected to the markets and following its own-arguably irregular-path. The GSADF also provides evidence of a bubble during the bicoin boom of 2013 before the Mt. Gox trading suspension and filing for bankruptcy protection.

Figure 1 presents the rolling window PC-LASSO coefficient estimates (with market/factor type components) for the ten independent variables whose coefficients are non-zero the most times in the rolling window estimation. ${ }^{9}$ Three of the ten variables are related to uncertainty (two economic policy uncertainty and one stock market uncertainty), three to stock market returns, and two are the internet trends. Notice that both in the early phase of lower traded quantities and the first bitcoin boom and in the third one we have identified, corresponding to the recent alleged bubble, the coefficients of Google and Wikipedia trends are generally positive (or zero), while in the middle phase (of the burst of the first boom and following Mt. Gox's suspension of trading and filing for bankruptcy protection), the coefficients turn more negative. This points to the capability of internet trends to accelerate the creation and the burst of a bubble. ${ }^{10}$

\section{Conclusion}

In this study we examine fourty-one potential drivers of bitcoin returns for the period 2010-2018, including stock market returns, stock market volatility, exchange rates, commodities, central bank rates, internet trends and policy uncertainty. We split the sample into the three different phases the bitcoin market has been

\footnotetext{
${ }^{9}$ Rolling window LASSO estimation has been employed by Li and Chen (2014), while Kapetanios and Zikes (2018) present an alternative methodology for time-varying Lasso estimation. Both of these are based on standard LASSO methods not exploiting the correlation and group structure of the independent variables through principal component guidance.

${ }^{10}$ Similar evidence is traced in the results in Tables 2 and 3.
} 
Table 2: PC-LASSO with market/factor type components coefficients of independent variables

\begin{tabular}{lcccc}
\hline Variable & Full sample & 1st period & 2nd period & 3rd period \\
\hline CBOE SPX Volatility VIX & -0.0358 & -0.0290 & 0.0000 & 0.0000 \\
US Equity-related EPU & 0.0242 & 0.0010 & 0.0000 & 0.0000 \\
Russia EPU & -0.0228 & -0.0062 & 0.0000 & 0.0000 \\
Australia EPU & -0.0215 & -0.0488 & 0.0000 & 0.0000 \\
Japan Government Benchmark Bid Yield & 0.0170 & 0.0000 & 0.0000 & 0.0000 \\
S\&P 500 Composite 2-day return & 0.0114 & 0.0098 & 0.0000 & 0.0000 \\
US Government Benchmark Bid Yield & 0.0026 & 0.0000 & 0.0000 & 0.0000 \\
South Korea EPU & 0.0020 & 0.0000 & 0.0000 & 0.0000 \\
ECB Overnight Deposit Rate & 0.0019 & 0.0000 & 0.0000 & - \\
MSCI Europe Minimum Volatility Index & 0.0015 & 0.0000 & 0.0000 & 0.0000 \\
Google Trend log difference & 0.0010 & 0.0000 & -0.0102 & 0.0000 \\
Euro Stoxx 50 Volatility Index & 0.0008 & 0.0000 & 0.0000 & 0.0000 \\
Nikkei Stock Average Volatility Index & -0.0004 & -0.0026 & 0.0000 & 0.0000 \\
Dow Jones 65 Composite 2-day return & 0.0000 & 0.0002 & 0.0000 & 0.0000 \\
US FFER & 0.0000 & 0.0000 & 0.0000 & -0.0074 \\
India EPU & 0.0000 & -0.0023 & 0.0000 & 0.0000 \\
Nikkei 225 Psychological Index & 0.0000 & -0.0071 & 0.0032 & 0.0000 \\
China Government Benchmark Bid Yield & 0.0000 & 0.0015 & -0.0263 & 0.0000 \\
US EPU & 0.0000 & -0.0046 & 0.0000 & 0.0000 \\
USD/UK pound return & 0.0000 & 0.0000 & -0.0049 & 0.0000 \\
Wikipedia trend log difference & 0.0000 & 0.0000 & -0.0026 & 0.0000 \\
\hline Cross-validation $\lambda$ & 39 & 21.06 & 56.42 & 53.87 \\
\hline Notes: the Chinese cental bank & &
\end{tabular}

Notes: the Chinese central bank rate does not vary in the 2 nd and $3 r d$ periods and the ECB rate does not vary in the 3rd period, so they have been excluded in the corresponding periods. Otherwise, all variables have been included in the regressions but the ones whose coefficients are zero in all three periods are not presented in the table. Principal component groups are as in the market/factor type column of Table 4. $\lambda$ for the total sample has been selected through cross-validation. Cross-validation has also been performed for each of the three sub-periods and then for comparability the average of the three resulting $\lambda$ 's has been used for all three sub-periods. The variables have been standardized in the full sample and each sub-period separately. Due to the penalties used in the estimation, the method gives no confidence intervals for the coefficients. 
Table 3: PC-LASSO with geographic region components coefficients of independent variables

\begin{tabular}{lcccc}
\hline Variable & Full sample & 1st period & 2nd period & 3rd period \\
\hline Russia EPU & -0.0032 & -0.0075 & 0.0000 & 0.0000 \\
CBOE SPX Volatility VIX & -0.0024 & -0.0097 & 0.0000 & 0.0000 \\
S\&P 500 Composite 2-day return & 0.0001 & 0.0040 & 0.0000 & 0.0000 \\
Australia EPU & 0.0000 & -0.0164 & 0.0000 & 0.0000 \\
Dow Jones 65 Composite 2-day return & 0.0000 & 0.0025 & 0.0000 & 0.0000 \\
US FFER & 0.0000 & 0.0000 & 0.0000 & -0.0010 \\
Google Trend log difference & 0.0000 & 0.0000 & -0.0094 & 0.0000 \\
India EPU & 0.0000 & -0.0099 & 0.0000 & 0.0000 \\
Japan Uncollateralized Overnight Rate & 0.0000 & 0.0000 & -0.0003 & 0.0000 \\
Japan EPU & 0.0000 & -0.0095 & 0.0000 & 0.0000 \\
Nikkei Stock Average Volatility Index & 0.0000 & -0.0043 & 0.0000 & 0.0000 \\
Singapore EPU & 0.0000 & -0.0042 & 0.0000 & 0.0000 \\
China Government Benchmark Bid Yield & 0.0000 & 0.0005 & -0.0025 & 0.0000 \\
Japan Government Benchmark Bid Yield & 0.0000 & 0.0000 & -0.0006 & 0.0000 \\
US EPU & 0.0000 & -0.0050 & 0.0000 & 0.0000 \\
USD/UK pound return & 0.0000 & 0.0000 & -0.0002 & 0.0000 \\
\hline Cross-validation $\lambda$ & 98.89 & 21.06 & 81.86 & 53.87 \\
\hline Notes: the Chinese central bank rate does
\end{tabular}

Notes: the Chinese central bank rate does not vary in the 2 nd and $3 r d$ periods and the ECB rate does not vary in the 3rd period, so they have been excluded in the corresponding periods. Otherwise, all variables have been included in the regressions but the ones whose coefficients are zero in all three periods are not presented in the table. Principal component groups are as in the region column of Table 4. Australia is grouped with Asia and the five variables ascribed to two regions are included in the groups of both regions. $\lambda$ for the total sample has been selected through cross-validation. Cross-validation has also been performed for each of the three sub-periods and then for comparability the average of the three resulting $\lambda$ 's has been used for all three sub-periods. The variables have been standardized in the full sample and each sub-period separately. Due to the penalties used in the estimation, the method gives no confidence intervals for the coefficients. 
Figure 1: Rolling window PC-LASSO coefficients with market/factor type components

(a) Five most-often non-zero coefficient

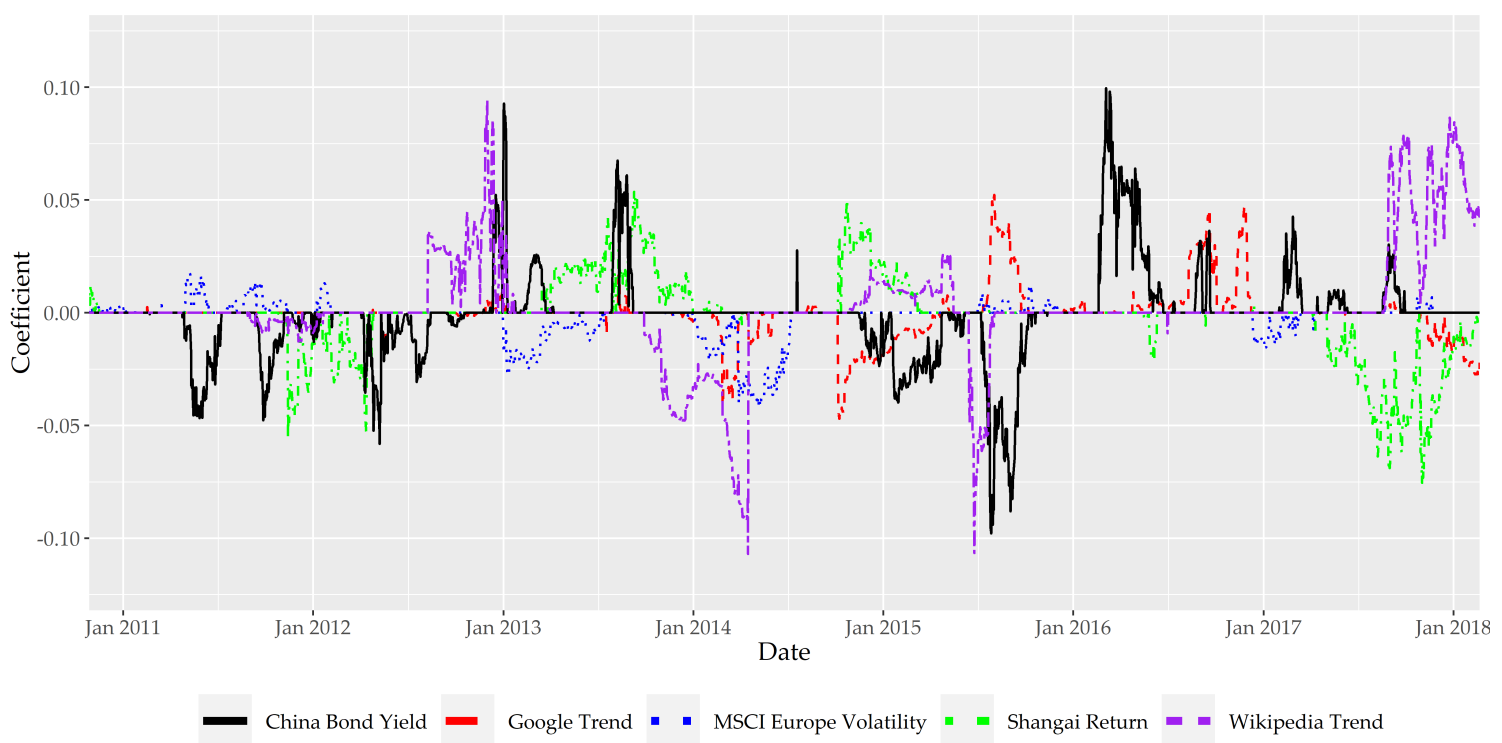

(b) Second five most-often non-zero coefficients

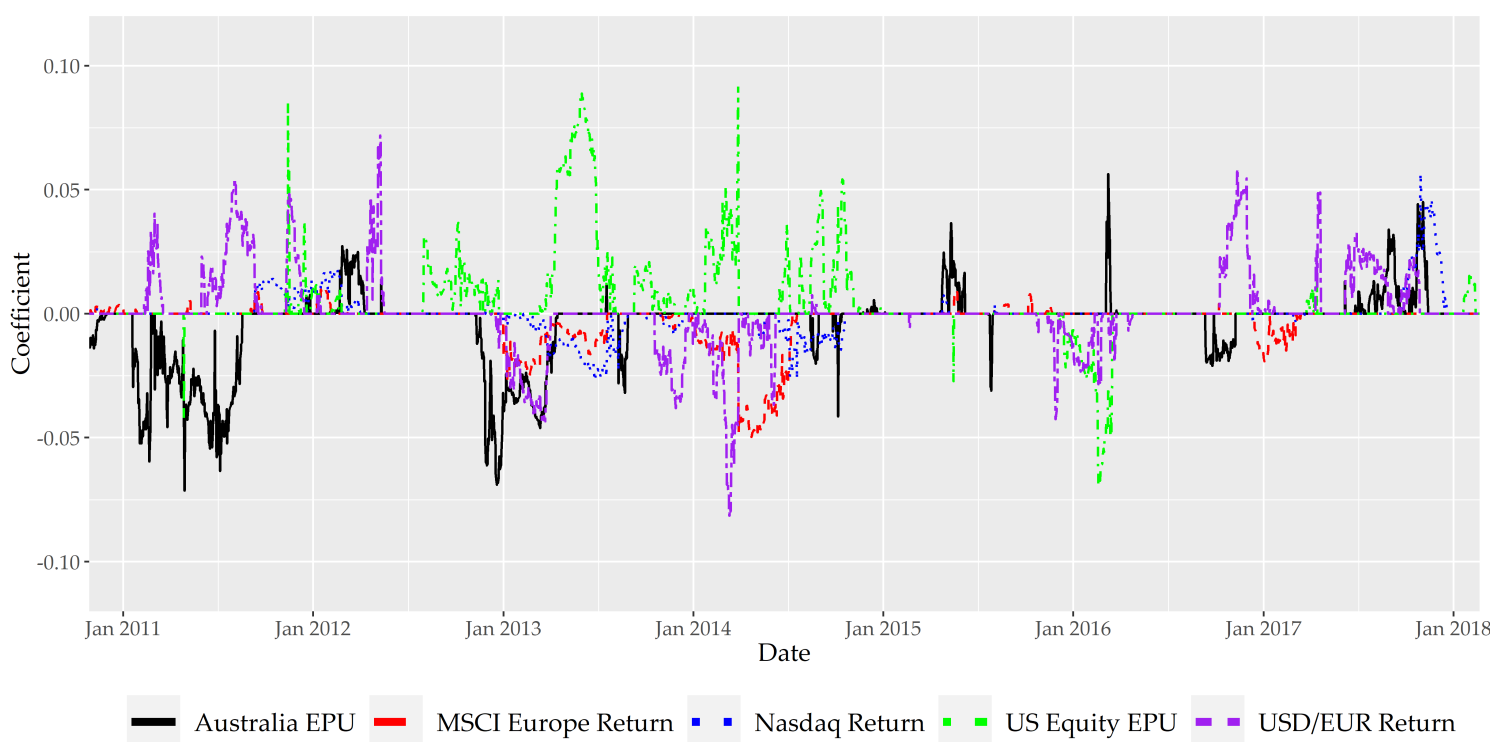

Note: the window size is 200 observations. All variables are standardized in each window separately. For comparability $\lambda=15$ and $r=0.7$ across all windows. The ECB the Chinese central bank rates have not been included in the analysis as they often do not vary within windows. The coefficients on a given date correspond to the window whose 100th observation corresponds to that date. In the first panel are the coefficients of the five independent variables whose coefficients are most often non-zero in the rolling window estimation (for example, the China bond yield is the variable whose coefficient is non-zero the most times, that is in 994 out of the 2,673 rolling window estimations); in the second panel are the five variables with the next highest numbers of non-zero coefficients. MSCI Europe volatility and USD/EUR return have equal numbers of non-zero coefficients (892). 
through based also on the rolling window GSADF test for bubbles (Phillips et al., 2015). Employing the principal component-guided sparse regression (PC-LASSO) recently introduced by Tay et al. (2018) we identify the variables that are most important for bitcoin. Furthermore, after selecting a subset of the examined variables based on the PC-LASSO results, we employ the Flexible Least Squares (FLS) methodology of Kalaba and Tesfatsion (1989) to gauge how the importance of alternate variables for bitcoin has changed over time.

We find that variables such as economic policy uncertainty and stock market volatility are among the most important ones for bitcoin. We also trace strong evidence of bubbly bitcoin behavior, especially in the 2017-2018 period, as well as evidence that internet trends can expedite the creation and the burst of a bubble. We find a minor importance of FX markets and monetary policy, but higher of traditional stock market returns. Commodity markets appear not to play a role, while the opposite holds for government bond yields. 


\section{REFERENCES}

Caspi, I. (2017). Rtadf: Testing for bubbles with eviews. Journal of Statistical Software, Code Snippets, 81(1):1-16.

Corbet, S., Lucey, B., and Yarovaya, L. (2018). Datestamping the Bitcoin and Ethereum bubbles. Finance Research Letters, 26:81-88.

Forbes, K. J. and Rigobon, R. (2002). No contagion, only interdependence: Measuring stock market comovements. Journal of Finance, 57(5):2223-2261.

Hotz-Behofsits, C., Huber, F., and Zörner, T. O. (2018). Predicting crypto-currencies using sparse non-Gaussian state space models. Journal of Forecasting, 37(6):627640.

Kalaba, R. and Tesfatsion, L. (1989). Time-varying linear regression via flexible least squares. Computers \& Mathematics with Applications, 17(8):1215 - 1245.

Kapetanios, G. and Zikes, F. (2018). Time-varying Lasso. Economics Letters, 169:1 6.

Li, J. and Chen, W. (2014). Forecasting macroeconomic time series: Lasso-based approaches and their forecast combinations with dynamic factor models. International Journal of Forecasting, 30(4):996 - 1015.

Nakamoto, S. (2008). Bitcoin: A peer-to-peer electronic cash system. Available at: https://bitcoin.org/bitcoin.pdf.

Panagiotidis, T., Stengos, T., and Vravosinos, O. (2018a). On the determinants of bitcoin returns: A LASSO approach. Finance Research Letters, 27:235-240.

Panagiotidis, T., Stengos, T., and Vravosinos, O. (2018b). The effects of markets, uncertainty and search intensity on bitcoin returns. International Review of Financial Analysis.

Phillips, P. C., Shi, S., and Yu, J. (2015). Testing for multiple bubbles: Limit theory of real-time detectors. International Economic Review, 56(4):1079-1134.

Phillips, P. C., Wu, Y., and Yu, J. (2011). Explosive Behavior In The 1990s Nasdaq: When Did Exuberance Escalate Asset Values?*. International Economic Review, 52(1):201-226.

Tay, J. K., Friedman, J., and Tibshirani, R. (2018). Principal component-guided sparse regression. arXiv e-prints, page arXiv:1810.04651. 


\section{OnLINE ApPEndix}

\section{A. The PC-LASSO Methodology}

This section follows Tay et al. (2018). Let $Y$ and $X$ be the vector and matrix of the dependent and the independent variables with centered columns, respectively. Also, let the $p$ potential predictors of bitcoin be grouped in $K$ non-overlapping groups. For $k=1, \ldots, K \boldsymbol{X}_{k}$ denotes the $p_{k}$ columns of $\boldsymbol{X}$ corresponding to group $k, m_{j}:=\operatorname{rank}\left(\boldsymbol{X}_{k}\right)$, and $\left(\boldsymbol{V}_{k}, d_{k}\right)$ denotes the right singular vectors and singular values of $\boldsymbol{X}_{k}$. PC-LASSO minimizes:

$$
J(\beta)=\frac{1}{2}\|\boldsymbol{y}-\boldsymbol{X} \beta\|_{2}^{2}+\lambda\|\beta\|_{1}+\frac{\theta}{2} \sum_{k} \beta_{k}^{T}\left(\boldsymbol{V}_{k} \boldsymbol{D}_{d_{k 1}^{2}-d_{k j}^{2}} \boldsymbol{V}_{k}^{T}\right) \beta_{k},
$$

where $\beta_{k}$ is the sub-vector of $\beta$ corresponding to group $k, d_{k}=\left(d_{k 1}, \ldots, d_{k m}\right)$ are the singular values of $\boldsymbol{X}_{k}$ in decreasing order, and $\boldsymbol{D}_{d_{k 1}^{2}-d_{k j}^{2}}$ is a diagonal matrix with diagonal entries $d_{k 1}^{2}-d_{k j}^{2}$ for $j=1, \ldots, m_{k} . \theta$ and $\lambda$ are parameters to be chosen, often through cross-validation. PC-LASSO gives:

$$
\boldsymbol{X} \widehat{\beta}=\sum_{j=1}^{m} \frac{d_{j}^{2}}{d_{j}^{2}+\theta\left(d_{1}^{2}-d_{j}^{2}\right) u_{j} u_{j}^{T} \boldsymbol{y}}
$$

where $m:=\operatorname{rank}(\boldsymbol{X}), u_{j}$ the $j$-th column of the matrix $\boldsymbol{U}$, where $\boldsymbol{U} \boldsymbol{D} \boldsymbol{V}^{T}$ the singular value decomposition of $\boldsymbol{X}$, and $d_{1} \geq d_{2} \geq \cdots \geq d_{m}>0$ the diagonal entries of the diagonal matrix $\boldsymbol{D} \cdot d_{j}^{2} /\left[d_{j}^{2}+\theta\left(d_{1}^{2}-d_{j}^{2}\right) u_{j} u_{j}^{T} \boldsymbol{y}\right]$ is called the shrinkage factor.

The objective function can be optimized efficiently by a coordinate descent procedure, since it is convex and the non-smooth component is separable. As the parameter $\theta$ is difficult to interpret, one can specify the ratio $r$ between the shrinkage factors in (1) for $k=2$ and $k=1$ (the latter being equal to 1 ). The admissible range for the ratio is $[0,1]$, where 1 corresponds to $\theta=0$ (standard LASSO) and lower values induce stronger shrinkage.

\section{B. DATA DESCRIPTION AND SUPPLEMENTARY RESULTS}

In Figure 3, panel (a) presents the FLS coefficient paths for the independent variables whose coefficients vary out of the thirteen variables with non-zero coefficients in the Full sample column of Table 2 included in the model. The ECB Overnight Deposit Rate appears significant in the beginning of the European debt crisis, in which period Europe stock market volatility also seems to have increased 
Figure 2: Coindesk BPI and the generalized sup augmented Dickey-Fuller statistic

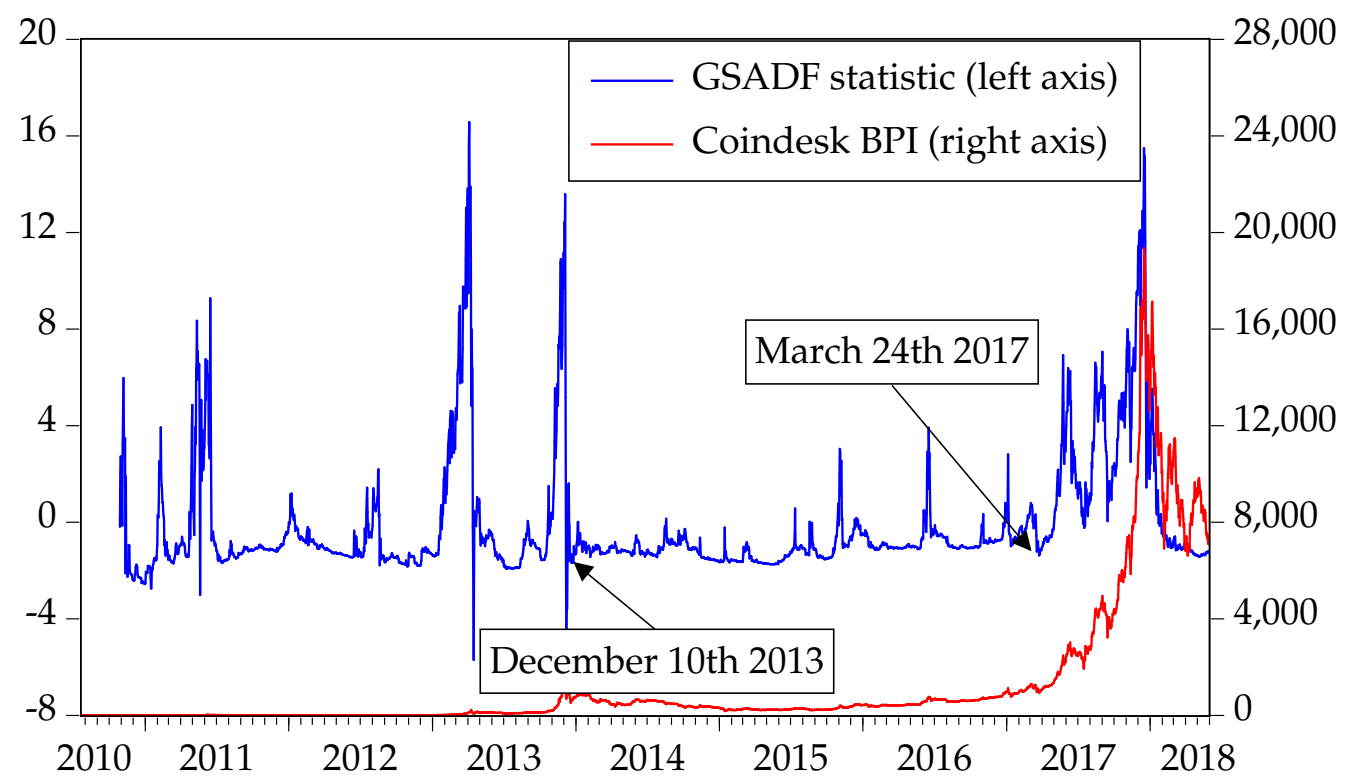

Notes: the final GSADF statistic is the largest ADF statistic, which in the figure is above 16 . The asymptotic $99 \%$ critical value for an initial window $5.5 \%$ (ours is 3.5\%) of the total sample is 2.74 based on numerical simulations with 2000 replications (Phillips et al., 2015). Given that the test statistic exceeds this value in some point (actually multiple) in the sample, there is evidence of bubbly behavior.

importance. Also, the US equity-related EPU appears constantly significant across the examined period. Panel (b) presents analogous results for a lower smoothing parameter value along with the coefficient paths under a standard state-space approach with Kalman filtering where coefficients are treated as separate random walks. The paths for the coefficients that vary over time are similar in the two methods. 
Figure 3: Flexible Least Squares and state-space varying coefficient paths

(a) Flexible Least Squares with Kalman filtering varying coefficient paths
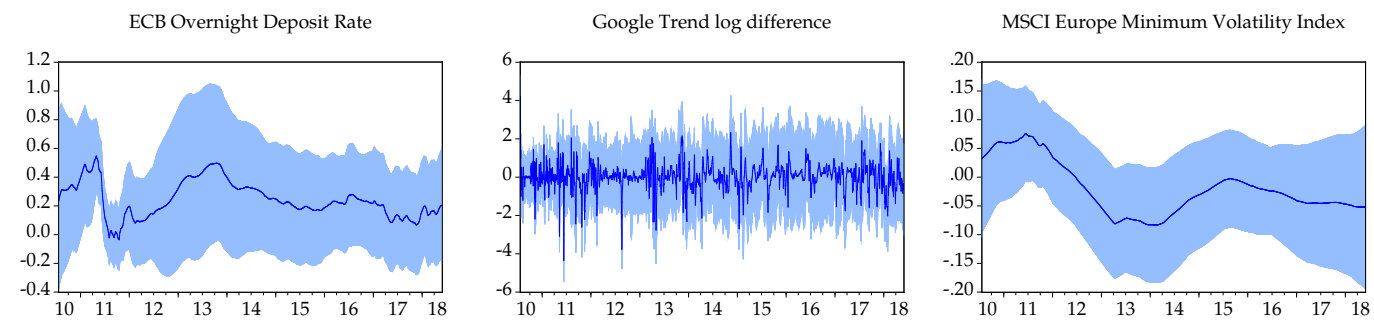

(b) Flexible Least Squares with Kalman filtering (in blue solid lines) and state-space with Kalman filtering (in red dashed lines) varying coefficient paths
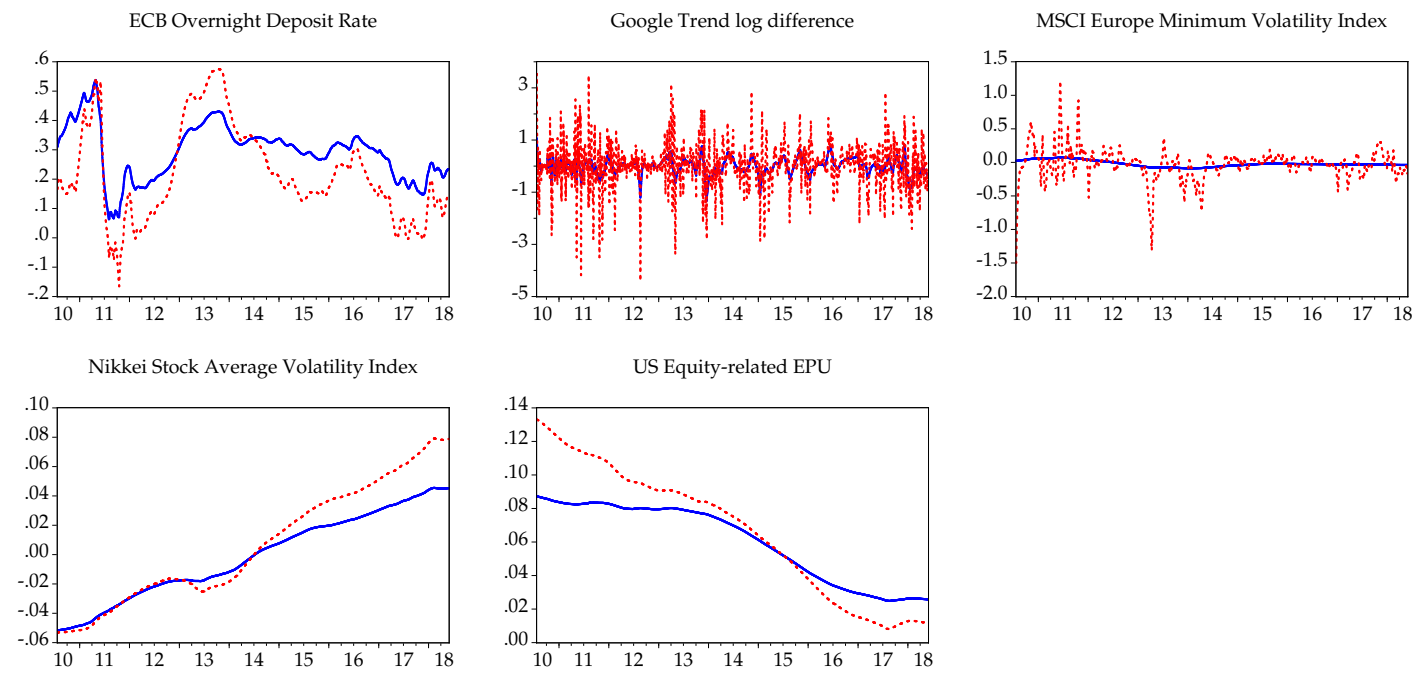

Notes: in panel (a) the smoothing parameter has been set to $1 ; \pm 2$ standard deviation bands are in light blue. In panel (b) the smoothing parameter for the FLS has been set to 0.1. 
Table 4: Variables employed, sample: June 21st 2010 to May 31st 2018 (7-day week; 2,872 observations)

\begin{tabular}{|c|c|c|c|}
\hline Variable & Market/factor type & Region & Eikon code \\
\hline Crude Oil-WTI Spot Cushing (in USD/BBL) & Commodities market returns & World & CRUDOIL \\
\hline MLCX - Gas oil Spot Index - price index & Commodities market returns & World & MLCXQSS \\
\hline S\&P GSCI Gold Total Return & Commodities market returns & World & GSGCTOT \\
\hline DJGL World - price index & Equity market returns & World & DJWRLD\$ \\
\hline Dow Jones 65 Composite Average - price index & Equity market returns & US & DJCMP65 \\
\hline MSCI Europe - price index & Equity market returns & Europe & MSEROP\$ \\
\hline Nasdaq Composite - price index & Equity market returns & US & NASCOMP \\
\hline Nikkei 225 Psychological - price index & Equity market returns & Asia & JAPDOWP \\
\hline Nikkei 225 Stock Average - price index & Equity market returns & Asia & JAPDOWA \\
\hline S\&P 500 Composite - price index & Equity market returns & US & S\&PCOMP \\
\hline Shangai SE Composite - price index & Equity market returns & Asia & CHSCOMP \\
\hline CBOE SPX Volatility VIX - price index & Equity market volatility & US & CBOEVIX \\
\hline Euro Stoxx 50 Volatility Index - price index & Equity market volatility & Europe & VST1MEI \\
\hline MSCI Europe Minimum Volatility (in USD) - price index & Equity market volatility & Europe & MSURMV\$ \\
\hline Nikkei Stock Average Volatility Index - price index & Equity market volatility & Asia & VXJINDX \\
\hline Chinese Yuan to USD (WMR) - exchange rate & FX market returns & US/Asia & CHIYUA\$ \\
\hline Japanese Yen to USD (WMR) - exchange rate & FX market returns & US/Asia & JAPAYE\$ \\
\hline USD to Euro (WMR\&DS) - exchange rate & FX market returns & US/Europe & USEURSP \\
\hline USD to UK pound (WMR) - exchange rate & FX market returns & US/Europe & USDOLLR \\
\hline China Government Benchmark Bid Yield - 10 Years & Government bond yields & Asia & TRCH10T \\
\hline Japan Government Benchmark Bid Yield - 10 Years & Government bond yields & Asia & TRJP10T \\
\hline US Government Benchmark Bid Yield - 10 Years & Government bond yields & US & TRUS10T \\
\hline Google Trend for the term "bitcoin" & Investor attention & World & - \\
\hline Wikipedia trend for the article on bitcoin & Investor attention & World & - \\
\hline Euro Overnight Deposit (ECB) - middle rate & Monetary policy & Europe & EURODEP \\
\hline Japan Uncollateralized Overnight - middle rate & Monetary policy & Asia & JPCALLO \\
\hline Chinese Renminbi 1D Notice Deposit - middle rate & Monetary Policy & Asia & CHDEPCL \\
\hline US Fed Funds Effective Rate - middle rate & Monetary policy & US & FRFEDFD \\
\hline Australia Economic Policy Uncertainty Index (news based) & Policy uncertainty & Australia & AUEPUNEWR \\
\hline China Economic Policy Uncertainty Index (news based) & Policy uncertainty & Asia & CHEPUNEWR \\
\hline EU Economic Policy Uncertainty Index (news based) & Policy uncertainty & Europe & EUEPUNEWR \\
\hline India Economic Policy Uncertainty Index (news based) & Policy uncertainty & Asia & INEPUNEWR \\
\hline Japan Economic Policy Uncertainty Index (news based) & Policy uncertainty & Asia & JPEPUOVAR \\
\hline Russia Economic Policy Uncertainty Index (news based) & Policy uncertainty & Europe/Asia & RSEPUNEWR \\
\hline Singapore Economic Policy Uncertainty Index (news based) & Policy uncertainty & Asia & SPEPUTWAR \\
\hline South Korea Economic Policy Uncertainty Index (news based) & Policy uncertainty & Asia & KOEPUOVAR \\
\hline UK Economic Policy Uncertainty Index & Policy uncertainty & Europe & UKEPUPO \\
\hline US Economic Policy Uncertainty Index (news based) & Policy uncertainty & US & USEPUNEWR \\
\hline US Economic Policy Uncertainty Index & Policy uncertainty & US & USEPUPO \\
\hline US Equity-related Economic Policy Uncertainty Index & Policy uncertainty & US & USEPUEQ \\
\hline World Economic Policy Uncertainty Index - PPP-adj & Policy uncertainty & World & WDEPUPPPR \\
\hline
\end{tabular}

